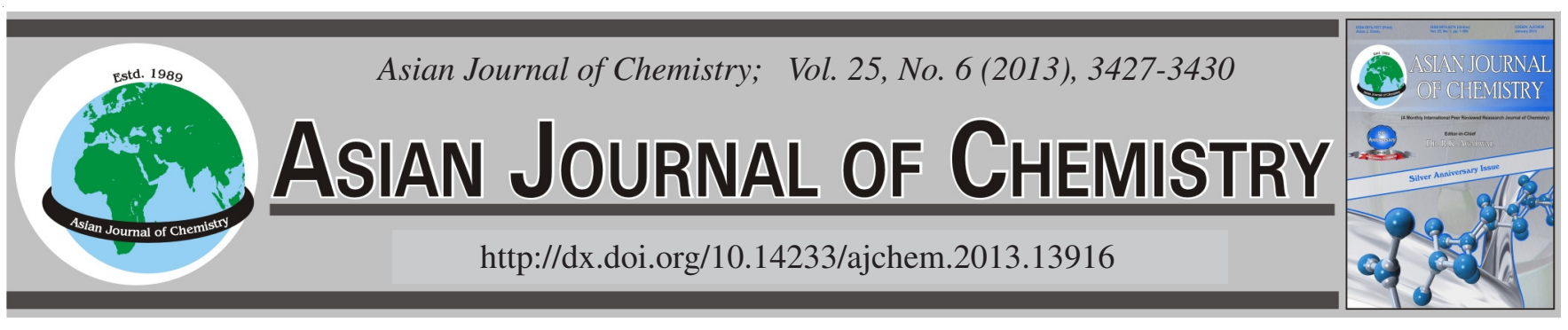

\title{
Nano Photocatalytic Process Application of ZnO Nanoparticle and UV on Benzene Removal from Synthetic Air
}

\author{
H.R. NASSEHINIA ${ }^{1}$, M. GHOLAmi ${ }^{1, *}$, A. JONIDI JAFARI ${ }^{2}$ and A. ESRAFILY ${ }^{3}$
}

${ }^{1}$ Department of Environmental Health Engineering, School of Public Health and Institute of Public Health Research, Tehran University of Medical Sciences, P.O. Box: 6446-14155, Tehran, Iran

${ }^{2}$ Department of Enivironmental Health, Faculty of Medical Sciences, Tarbiat Modares University, Tehran, Iran

${ }^{3}$ Department of Chemistry, Tarbiat Modares University, Tehran, Iran

*Corresponding author: E-mail: gholamim@ tums.ac.ir

Ornament painting and insects repellent solvents based on organic compounds, especially benzene compounds, were widely used in recent years. Exposure to gaseous benzene causes carcinogen and acute and chronic skin disease and even adverse effects on the nervous system. Photocatalytic systems are suitable for treating low concentration pollutants in air. The focus of this paper is on the photocatalytic degradation of benzene in photo reactor of $\mathrm{ZnO}$ catalyst by ultra violet radiation. The conventional sol-gel method was employed for the preparation of $\mathrm{ZnO}$ catalyst. The prepared $\mathrm{ZnO}$ sol-gel was coated by thin film dip-coating to deposit the catalyst on glass. UV lamps with a wavelength peak at $365 \mathrm{~nm}$ was employed as UV light source. ZnO thin film increased the photocatalytic activity due to the maximization of UV lamps illumination and increasing of retention time of benzene in the reactor. In this research, removal of benzene by $\mathrm{ZnO}$ and UV follows the zero order reactions.

Key Words: Photocatalytic, Benzene, ZnO, Ultra violet.

ᄂ - - - - - - - - - - - - - - - - - - - - - - - - $\lrcorner$

\section{INTRODUCTION}

Volatile organic carbon compounds have an important effect in the chemistry of the atmosphere. Their role in the formation of photochemical oxidants, threatening both human and ecosystem health and their contribution to stratospheric ozone depletion and the greenhouse effect are well known ${ }^{1}$.

Ornament painting and insects repellent solvents based on organic compounds, especially benzene compounds, were widely used in recent years and then the gaseous pollutants can be released into environments through slow diffusion and have resulted in increased levels of air pollution. Exposure to gaseous benzene causes carcinogen and acute and chronic skin disease and even adverse effects on the nervous system ${ }^{2}$.

Conventional methods used to removal volatile organic carbons include incineration, condensation, adsorption and absorption. Incineration and condensation are usable only for moderate to high volatile organic carbon concentrations. Adsorption and absorption do not destroy volatile organic carbons but transfer them to another medium. None of these methods is economical for gas streams with low to moderate concentration of pollutants.
Photocatalytic systems do not have the above problems and are suitable for treating low concentration pollutants ${ }^{3}$. $\mathrm{ZnO}$ as a potential photocatalyst has been widely investigated in recent years ${ }^{4,5}$. Benzene is the one of organic compounds that have been studied in heterogeneous gas-phase $\mathrm{PCO}^{6-8}$.

\section{EXPERIMENTAL}

It has been shown that benzene is less susceptible to photocatalytic oxidation than toluene. Reactivities chlorine radical and hydroxyl radical have been studied with toluene and benzene and was found that toluene is 13 times more reactive than benzene with chlorine radicals, but much closer reactivities between toluene and benzene were observed with only hydroxyl radicals present ${ }^{9}$. The difference could partially explain why the presence of chlorine does not enhance the degradation rate of benzene. Two routes have been proposed for benzene degradation:

1. (i) Direct hole oxidation followed by reaction of the resulting radical cation either(ii) with a surface basic OH group, or (iii) with an adsorbed water mole-cule and subsequent deprotonation to yield phenol, the major intermediate detected. 


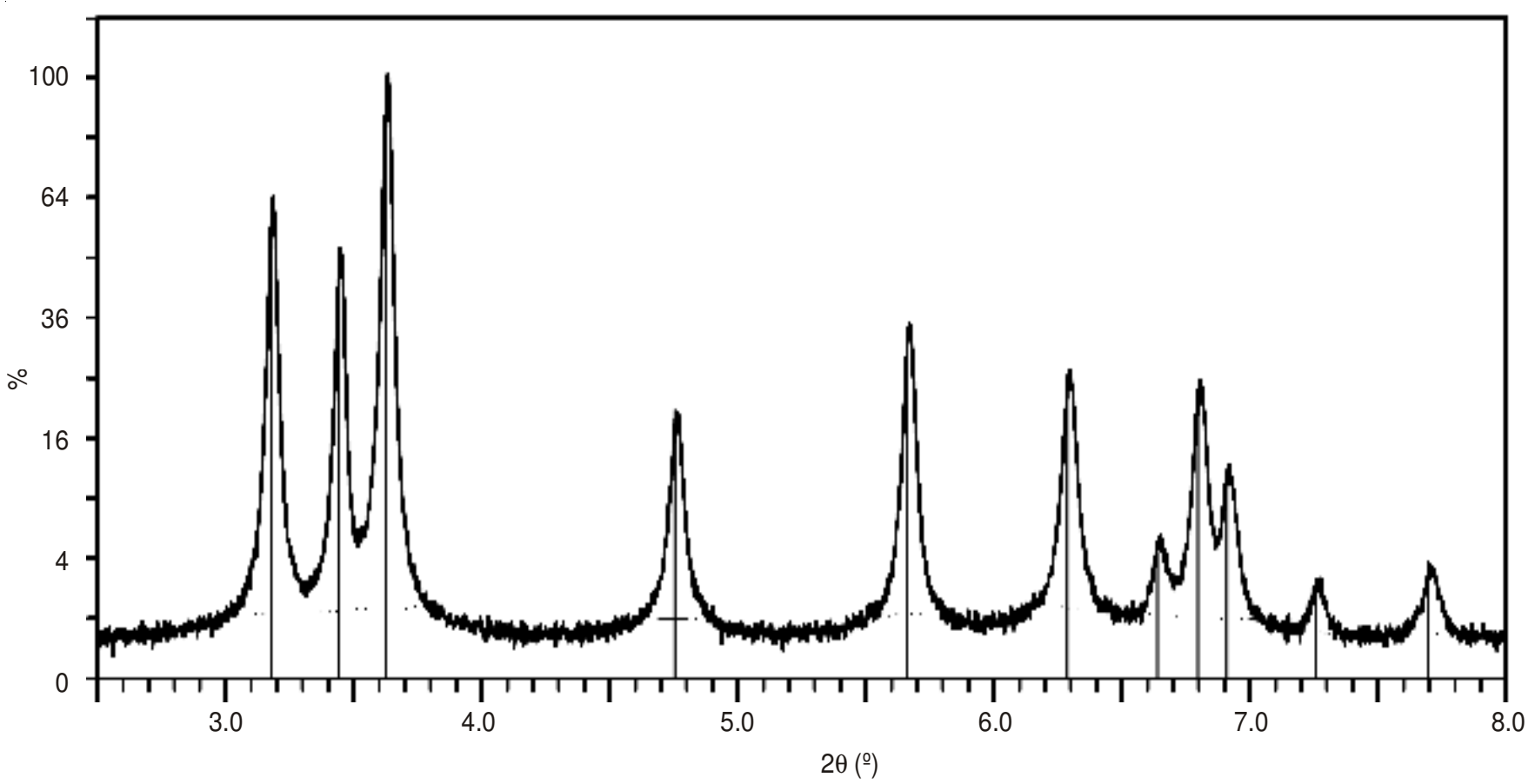

Fig. 1. XRD pattern of $\mathrm{ZnO}$ nano powders catalyst

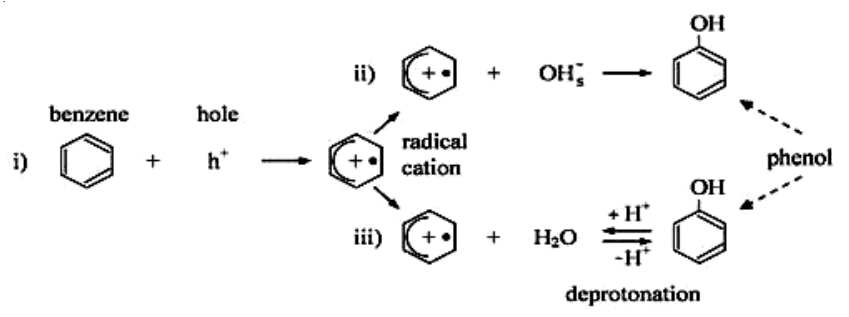

2. $\mathrm{OH}^{\bullet}$ radical addition to yield a cyclohexadienyl radical. Addition of oxygen to this radical and elimination of the radical $\mathrm{HO}_{2}{ }^{\bullet}$ produces phenol by analogy with what has been determined in water ${ }^{6,10}$.

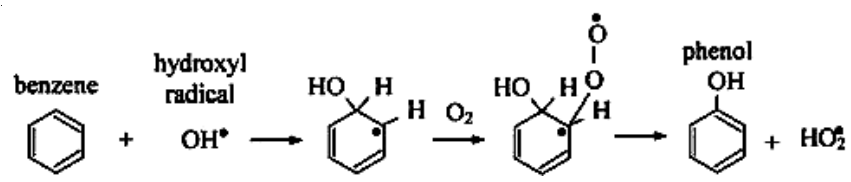

Though photocatalytic reactions can occur in both gas and liquid phases, the focus of this paper is on those reactions where gas-phase species are react on solid surfaces, referred to as heterogeneous (gas/solid) photocatalysis.

Catalyst preparation: The conventional sol-gel method was employed for the preparation of $\mathrm{ZnO}$ catalyst. Ethanol was used as a solvent. The $\mathrm{pH}$ value was fixed at 2 by $\mathrm{HNO}_{3}$ addition. Then, the solution was dispersed by ultrasonic technology in an ultrasonic cleaner at $200 \mathrm{~W}$ and $59 \mathrm{KHz}$ for $1 \mathrm{~h}$. The prepared $\mathrm{ZnO}$ sol-gel was coated by thin film dip-coating to deposit the catalyst on glass. After applying a layer of catalyst, the glass was dried in air at for $1 \mathrm{~h}$ to form solid $\mathrm{ZnO}$ layer and calcined in furnace at $500{ }^{\circ} \mathrm{C}$ for $0.5 \mathrm{~h}$. The structure of the catalysts and image of the $\mathrm{ZnO}$ film deposited on glass was measured by powder XRD and SEM, respectively. The photocatalytic degradation of benzene was investigated in photo reactor of $\mathrm{ZnO}$ catalyst. UV lamps with a wavelength peaking at $365 \mathrm{~nm}$ was employed as UV light source. The experiments were conducted at benzene concentration in polluted air of $100-300 \mu \mathrm{g} / \mathrm{L}$. The prepared catalyst was ground to powder and detected the crysta-lline properties of $\mathrm{ZnO}$ by an X-ray diffractometer. Surface morphology of the ordered $\mathrm{ZnO}$ porous thin film on the glass was determined by SEM. For the determination of benzene concentration in waste air, gas samples were taken from inlet (sampling1) and outlet (sampling 2) of the photo reactor using charcoal activated carbon adsorbent tube. Gas samples of $0 / 2 \mathrm{l} / \mathrm{min}$ and $1 \mathrm{~min}$ were acquired by sampling pump passing through adsorbent tube for every $0.5 \mathrm{~h}$ retention time of benzene. Desorption for the benzene sample was taken using carbon di solfide and then benzene concentration was analyzed using a gas chromatography model CP 9001, CHROM PACK company. The gas chromatography equipped with FID capillary column, a flame ionization detector and software $\mathrm{CP}$ chem stations was operated at injection temperature of $270^{\circ} \mathrm{C}$, detector temperature of $250^{\circ} \mathrm{C}$ and oven temperature of $40^{\circ} \mathrm{C}$. The gas chromatography column was $30 \mathrm{~m}$ capillary glass column with an inside diameter of $0.32 \mathrm{~mm}$ and film thickness of $0.25 \mathrm{~mm}$. Helium was used as the carrier gas at a flow rate of $1.5 \mathrm{~mL} / \mathrm{min}$. This analytical system gave an excellent resolution for benzene concentration measurement. The removal efficiency of the photocatalytic system was calculated by the following equation:

Benzene removal eff. $(\%)=\mathrm{C}_{\mathrm{in}}-\mathrm{C}_{\text {out }} / \mathrm{C}_{\text {in }} \times 100$ where, the $\mathrm{C}_{\text {in }}$ and $\mathrm{C}_{\text {out }}$ (ppm) are the benzene concentration observed at the inlet and outlet of photo reactor, respectively.

\section{RESULTS AND DISCUSSION}

The $\mathrm{ZnO}$ catalyst was prepared by the sol-gel method. In Fig. 1, shows the $\mathrm{X}$ ray diffraction patterns of the catalyst sample at calcinations temperature of $500{ }^{\circ} \mathrm{C}$. Fig. 2 shows the SEM images of ordered $\mathrm{ZnO}$ structures coated on glass 
substrates after calcination temperature of $500^{\circ} \mathrm{C}$. The SEM analysis shows the cracking of the thin film and complete covering of $\mathrm{ZnO}$ on the substrate.

Effect of photocatalyst illumination: A series of experiments were conducted at different light powers of UV lamp light sources. UV lamp powers was 4, 6 and $10 \mathrm{w} / \mathrm{m}^{2}$. Efficient illumination of the catalyst is a critical design feature within a plug flow photocatalytic reactor. Ideally catalyst packing should be transparent to UV light to allow activation of $\mathrm{ZnO}$ and degradation of contaminant substances. Fig. 3 is a plot of removal efficiency of benzene in different UV light powers without use of nano $\mathrm{ZnO}$. The figure reveals that the benzene degradation is very low with ultraviolet light without nano catalyst.

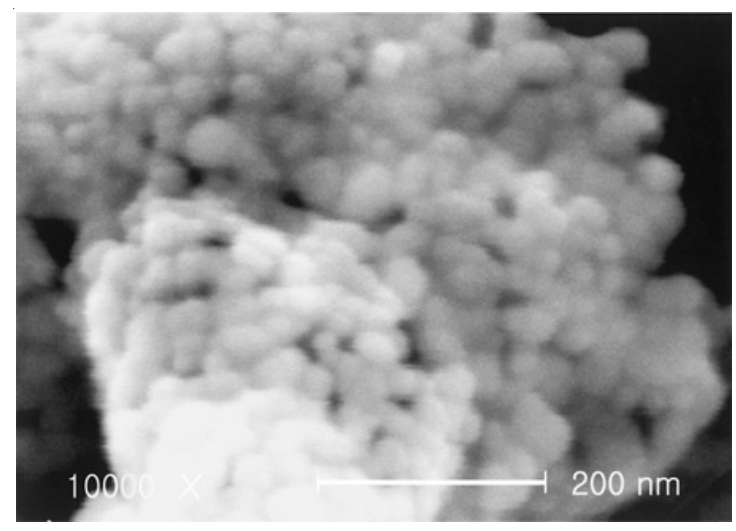

Fig. 2. SEM photograph of $\mathrm{ZnO}$ nanopowder

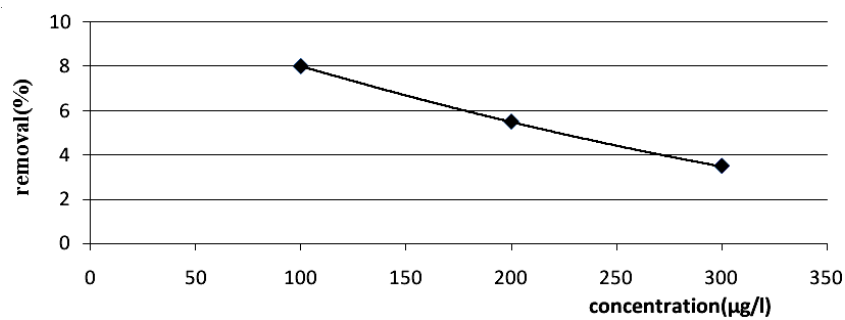

Fig. 3. Removal efficiency of benzene in ultraviolet light powers of 4,6,10 $\mathrm{w} / \mathrm{m}^{2}$ without $\mathrm{ZnO}$

Effect of photocatalyst loading: The catalytic material, $\mathrm{ZnO}$ thin film on glass, was packed in the photo reactor at $\mathrm{ZnO}$ loading quantity of $1.5 \mathrm{~g} / \mathrm{L}$. Fig. 4 represents experimental data of removal efficiency medium of benzene in different UV light powers with $\mathrm{ZnO}$. Fig. 4 reveals that the benzene degradation increases with use of catalyst and increase of ultraviolet lamp power. UV lamp installed in the photo reactor with power of $10 \mathrm{w} / \mathrm{m}^{2}$ provides the highest removal efficiency of $\mathrm{ZnO}$ in the waste air as its highest light power. From the results, it is obvious that the light power has a great effect on the photocatalytic reaction.

Fig. 5 shows kinetic reaction of benzene removal by $\mathrm{ZnO}$ and ultraviolet based on zero order reaction.

Quantity of $\mathrm{R}^{2}$ was calculated 0.9542 in Zero order reaction. Fig. 6 shows kinetic reaction of benzene removal by $\mathrm{ZnO}$ and UV based on first order reaction.

Quantity of $\mathrm{R}^{2}$ was calculated 0.8908 in first order reaction. Fig. 7 shows kinetic reaction of benzene removal by ZNO and ultraviolet based on first order reaction.

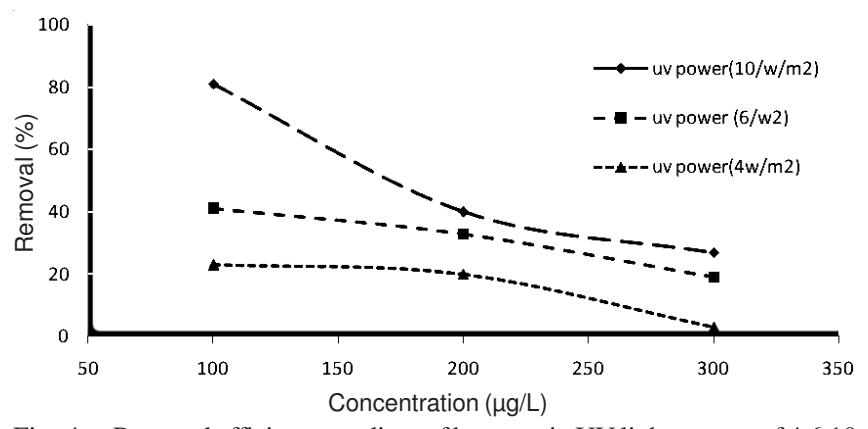

Fig. 4. Removal efficiency medium of benzene in UV light powers of 4,6,10 w/ $\mathrm{m}^{2}$ with $\mathrm{ZnO}$

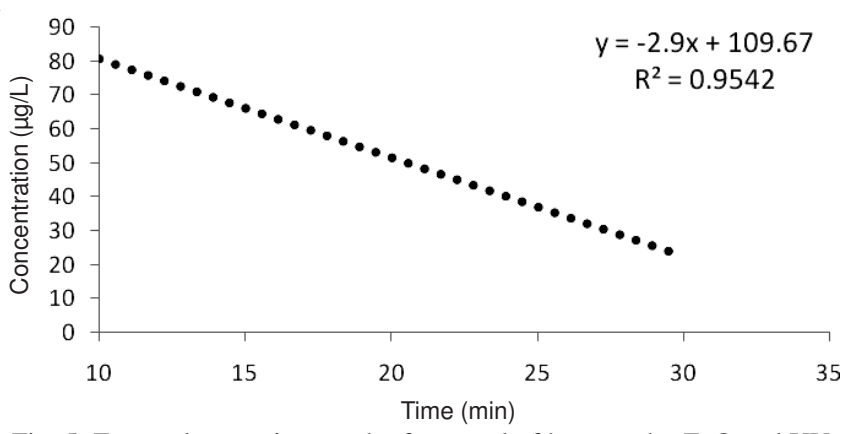

Fig. 5. Zero order reaction graph of removal of benzene by $\mathrm{ZnO}$ and UV

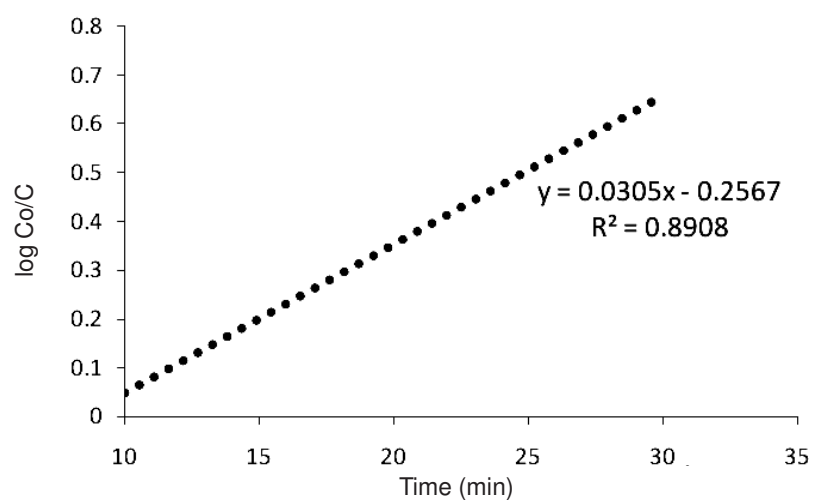

Fig. 6. First order reaction graph of benzene removal by $\mathrm{ZnO}$ and $\mathrm{UV}$

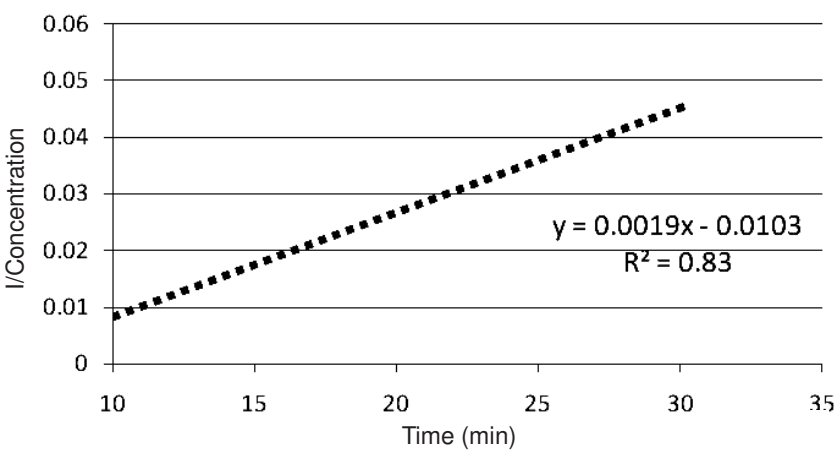

Fig. 7. Second order reaction graph of removal of benzene by $\mathrm{ZnO}$ and UV

Quantity of $\mathrm{R}^{2}$ was calculated 0.83 in second order reaction.

\section{Conclusion}

Benzene or benzol is a organic chemical compound that it is a carcinogen and it is a important solvent and is used in production of drugs, plastics and dyes ${ }^{11}$. In this study, composite $\mathrm{ZnO}$ catalyst was successfully prepared by sol-gel method to remove benzene in waste air. $\mathrm{ZnO}$ calcined at $500{ }^{\circ} \mathrm{C}$ 
indicated the mainly anatase crystalline form. The $\mathrm{ZnO}$ sol deposited on to glass was effectively performed to get thin film of photocatalyst. The studies of benzene removal from waste air in the reactor using the prepared $\mathrm{ZnO}$ were performed to compare the influence of illumination, photocatalyst packed loading. Oxidation of benzene on the photocatalyst was found to be significantly affected by all parameters. In research of photocatalytic characteristic was reported that benzene and toluene is degraded because of hydroxyl radicals existing ${ }^{10}$.

Fig. 4 showed that the $\mathrm{ZnO}$ photo degradation decreased when the initial benzene concentration was increased. Eighty one percent of benzene was degraded when its concentration in the reactor was $100 \mu \mathrm{g} / \mathrm{L}$ and power of $\mathrm{UV}$ was $10 \mathrm{w} / \mathrm{m}^{2}$, therefore meaning different existed (with $\alpha \leq 0.05$ ) between input and output concentration of benzene. Nevertheless, the removal obtained with concentration of $300 \mu \mathrm{g} / \mathrm{L}$ and $4 \mathrm{w} / \mathrm{m}^{2}$ UV power was only $3 \%$. In other research about removal of benzene with concentration of $100 \mu \mathrm{g} / \mathrm{L}$ in air concluded that benzene can completely removal with effect of ultra violet in wave length of $365 \mathrm{~nm}$ and $\mathrm{ZnO}$ photocatalyst $\mathrm{t}^{12}$.

As can be seen in Figs. 5-7, quantity of $\mathrm{R}^{2}$ calculated in zero order reaction graph is maximum, therefore in this research benzene removal by $\mathrm{ZnO}$ and ultraviolet follows from zero order reactions. Therefore, degradation of benzene will increase if concentration of benzene be decreased and power of ultraviolet lamp be increased.

\section{ACKNOWLEDGEMENTS}

This research was financially supported by Tehran University of Medical Sciences in Iran country. The authors thank from the Department of Health.

\section{REFERENCES}

1. R.R. Maria, M.M. Rosa and B. Francesc, J. Environ. Monit. Assess., 161, 1 (2010).

2. Q.-J. Geng, Q.-J. Guo and C.-Q. Cao and H.-Q. Wang, J. Chem. Eng. Technol., 31, 1023 (2008).

3. L. Zou, Y. Luo, M. Hooper and E. Hu, J. Chem. Eng. Process., 45, 959 (2006).

4. B. Dindar and O. Seven, Asian J. Chem., 21, 2270 (2009).

5. M.A. Hernandez, L. Corona and A. Gonzalez, J. Chem., 44, 9 (2005).

6. O. Hennezel, P. Pichat and D.F. Ollis, J. Photochem. Photobiol. A, 118, 3 (1998)

7. H. Einaga, S. Futamura and T. Ibusuki, Phys. Chem. Chem. Phys., 1, 4903 (1999).

8. A. Linsebigler, J.T. Lu and J.T. Yates, Chem. Rev., 95, 735 (1995).

9. O. Hennezel and D.F. Ollis, J. Catal., 167, 118 (1997).

10. D.T. Tompkins, B. Lawnicki and W.A. Zelter, www.fotokleen.net/pdf/ 5_3.pdf (2005).

11. W. Sung, K. Misook and S. Choung, J. Ind. Eng. Chem., 11, 3 (2005).

12. J. Peral, X. Domenech and D.F. Ollis, J. Chem. Tech. Biotechnol., 70, 117 (1997). 